\title{
CONVERGENCE OF SCALAR CURVATURE OF KÄHLER-RICCI FLOW ON MANIFOLDS OF POSITIVE KODAIRA DIMENSION
}

\author{
WANGJIAN JIAN
}

\begin{abstract}
In this paper, we consider Kähler-Ricci flow on n-dimensional Kähler manifold with semi-ample canonical line bundle and $0<m:=$ $\operatorname{Kod}(X)<n$. Such manifolds admit a Calabi-Yau fibration over its canonical model. We prove that the scalar curvature of the Kähler metrics along the normalized Kähler-Ricci flow converge to $-m$ outside the singular set of this fibration.
\end{abstract}

\section{INTRODUCTION}

Let us first recall the set up of Song-Tian [6, 7, 9] where our result will apply. Let $\left(X^{n}, \omega_{0}\right)$ be a compact Kähler manifold with canonical line bundle $K_{X}$ being semi-ample and $0<m:=\operatorname{Kod}(X)<n$. Therefore the canonical ring $R\left(X, K_{X}\right)$ is finitely generated, and so the pluricanonical system $\left|\ell K_{X}\right|$ for sufficiently large $\ell \in \mathbb{Z}^{+}$induces a holomorphic map

$$
f: X \rightarrow B \subset \mathbb{C P}^{N}:=\mathbb{P} H^{0}\left(X, K_{X}^{\otimes \ell}\right),
$$

where $B$ is the canonical model of $X$. We have $\operatorname{dim} B=m$.

Let $S^{\prime}$ be the singular set of $B$ together with the set of critical values of $f$, and we define $S=f^{-1}\left(S^{\prime}\right) \subset X$.

Now let $\omega(t)$ be the smooth global solution of the normalized Kähler-Ricci flow

$$
\frac{\partial \omega}{\partial t}=-\operatorname{Ric}(\omega)-\omega,\left.\omega\right|_{t=0}=\omega_{0}
$$

It's well-known [11, 15] that the flow has a global solution on $X \times[0, \infty)$. It's shown by Song-Tian [6, 7] that $\omega(t)$ collapses nonsingular Calabi-Yau fibers and the flow converges weakly to a generalized Kähler-Einstein metric $\omega_{B}$ on its canonical model $B$, with $\omega_{B}$ is smooth and satisfies the generalized Einstein equation on $B \backslash S^{\prime}$

$$
\operatorname{Ric}\left(\omega_{B}\right)=-\omega_{B}+\omega_{\mathrm{WP}}
$$

where $\omega_{\mathrm{WP}}$ is the Weil-Petersson metric induced by the Calabi-Yau fibration $f$. They also proved the $C^{0}$-convergence on the potential level and in the case when $X$ is an elliptic surface the $C_{l o c}^{1, \alpha}$-convergence of potentials on $X \backslash S$ for any $\alpha<1$. In 9 , Song-Tian showed that the scalar curvature is uniformly bounded on $X \times[0, \infty)$ along the normalized flow. The case 
when $X$ is of general type is given by Z.Zhang in [17]. The case for conical Kähler-Ricci flow is given by G.Ewards in [3].

In [2], Fong-Zhang proved the $C^{1, \alpha}$-convergence of potentials when $X$ is a global submersion over $B$ and showed the Gromov-Hausdorff convergence in the special case. In [13] Tosatti-Weinkove-Yang improved the estimate and showed that the metric $\omega(t)$ converges to $f^{*} \omega_{B}$ in the $C^{0}$ local-topology on $X \backslash S$. Moreover, Tosatti-Weinkove-Yang 13 proved that the restricted metric $\left.\omega(t)\right|_{X_{y}}$ converges (up to scalings) in the $C^{0}$-topology to the unique Ricci flat metric on the fibre $X_{y}$ for any regular value $y$; this result is improved to be smooth convergence by Tosatti-Zhang in [14. Also see Tosatti's note [12] for clearer and more unified discussions.

In fact, Tosatti-Weinkove-Yang [13] obtained in their proof that $\| \omega(t)-$ $\widetilde{\omega}(t) \|_{\omega(t)} \rightarrow 0$ as $t \rightarrow \infty$ on $X \backslash S$, where $\widetilde{\omega}(t)=e^{-t} \omega_{\text {SRF }}+\left(1-e^{-t}\right) \omega_{B}$ (see Section 2 for definition of $\left.\omega_{\mathrm{SRF}}\right)$. This enable us to prove that $\mid \operatorname{tr}_{\omega(t)} \omega_{B}-$ $m|+|\left\|\omega_{B}\right\|_{\omega(t)}^{2}-m \mid \rightarrow 0$ as $t \rightarrow \infty$ on $X \backslash S$, which then enable us to improve the estimate of scalar curvature on $X \backslash S$, following the argument of SongTian [9]. In this paper, we prove that the scalar curvature $R(t)$ converges on the regular part $X \backslash S$.

Theorem 1.1. Let $\left(X, \omega_{0}\right)$ be given as above, let $\omega(t)$ be the smooth global solution of the normalized Kähler-Ricci flow (1.2). Then we have

$$
\lim _{t \rightarrow \infty} R(t)=-m \text {, on } X \backslash S \times[0, \infty) .
$$

In particular, if $S=\emptyset$, then $f$ is a holomorphic submersion and we have

$$
|R(t)+m| \leqslant C e^{-\eta t}, \text { on } X \times[0, \infty),
$$

for some constants $\eta, C>0$ depending on $\left(X, \omega_{0}\right)$.

After rescaling time and space simultaneously, we have the following immediately corollary from Theorem 1.1 of the unnormalized Kähler-Ricci flow.

Corollary 1.2. Let $\left(X, \omega_{0}\right)$ be given as above, let $\omega(t)$ be the smooth global solution of the unnormalized Kähler-Ricci flow

$$
\frac{\partial \omega}{\partial t}=-\operatorname{Ric}(\omega),\left.\omega\right|_{t=0}=\omega_{0} .
$$

Then we have

$$
\lim _{t \rightarrow \infty}(1+t) R(t)=-m \text {, on } X \backslash S \times[0, \infty) .
$$

In particular, if $S=\emptyset$, then $f$ is a holomorphic submersion and we have

$$
|(1+t) R(t)+m| \leqslant \frac{C}{(1+t)^{\eta}} \text {, on } X \times[0, \infty),
$$

for some constants $\eta, C>0$ depending on $\left(X, \omega_{0}\right)$.

Note that in Theorem 1.1, the limiting behavior of scalar curvature on the singular set $S$ is unknown. A recent result of the author and two other authors [4] says that: If the canonical bundle $K_{X}$ is semi-ample, then for any 
Kähler class $[\omega]$ on $X$, there exists $\delta_{X,[\omega]}>0$ such that for any $0<\delta<\delta_{X,[\omega]}$, there exists a unique cscK metric in the Kähler class $\left[K_{X}\right]+\delta[\omega]$. Hence we can propose the following conjecture.

Conjecture 1.3. Let $X$ be an n-dimensional Kähler manifold with nef canonical bundle $K_{X}$ and positive Kodaira dimension. Then for any initial Kähler metric $\omega_{0}$, the solution $\omega(t)$ of the normalized Kähler-Ricci flow

$$
\frac{\partial}{\partial t} \omega=-\operatorname{Ric}(\omega)-\omega, \omega(0)=\omega_{0}
$$

converges in Gromov-Hausdorff topology to $\omega_{B}$ and the scalar curvature $R(t)$ converges to $-\operatorname{Kod}(X)$ in $C^{0}(X)$, where $\operatorname{Kod}(X)$ is the Kodaira dimension of $X$.

In general, it is natural to ask if the following holds for the maximal solution of the unnormalized Kähler-Ricci flow on $X \times[0, T)$, where $X$ is a Kähler manifold and $T>0$ is the maximal existence time.

(1) If $T<\infty$, then there exists $C>0$ such that

$$
-C \leqslant R(t) \leqslant C(T-t)^{-1} \text {. }
$$

(2) If $T=\infty$, then there exists $C>0$ such that

$$
|R(t)| \leqslant C(1+t)^{-1} \text {. }
$$

In [5], the answer to the first question is affirmative due to Perelman for the Kähler-Ricci flow on Fano manifolds with finite time extinction. In [18, it is shown that if the Kähler-Ricci flow develops finite time singularity, the scalar curvature blows up at most of rate $(T-t)^{-2}$ if $X$ is projective and if the initial Kähler class lies in $H^{2}(X, \mathbb{Q})$.

Acknowledgements. The author would like to thank his advisor Gang Tian for leading him to study Kähler-Ricci flow, constant encouragement and support. The author would like to thank Jian Song for helpful discussions. The author also would like to thank Yalong Shi and Dongyi Wei for helpful discussions. This work was carried out while the author was visiting Jian Song at the Department of Mathematics of Rutgers University, supported by the China Scholarship Council (File No.201706010022). The author would like to thank the China Scholarship Council for supporting this visit. The author also would like to thank Jian Song and the Department of Mathematics of Rutgers University for hospitality and support.

\section{Preliminary for the KÄHLer Ricci-Flow}

In this section let us recall some known results that we need in our proof.

From (1.1), we have $f^{*} \mathcal{O}(1)=K_{X}^{\otimes \ell}$, hence if we let $\chi=\frac{1}{\ell} \omega_{\mathrm{FS}}$ on $\mathbb{P} H^{0}\left(X, K_{X}^{\otimes \ell}\right)$, we have that $f^{*} \chi$ (later, denoted by $\chi$ ) is a smooth semipositive representative of $-c_{1}(X)$. Here, $\omega_{\mathrm{FS}}$ denotes the Fubini-Study metric. Also, we denote by $\chi$ the restriction of $\chi$ to $B \backslash S^{\prime}$. 
Given a Kähler metric $\omega_{0}$ on $X$, since $X_{y}:=f^{-1}(y)$ are Calabi-Yau for $y \in$ $B \backslash S^{\prime}$, there exists a unique smooth function $\rho_{y}$ on $X_{y}$ with $\int_{X_{y}} \rho_{y} \omega_{0}^{n-m}=0$, and such that $\left.\omega_{0}\right|_{X_{y}}+\sqrt{-1} \partial \bar{\partial} \rho_{y}=: \omega_{y}$ is the unique Ricci-flat Kähler metric on $X_{y}$. Moreover, $\rho_{y}$ depends smoothly on $y$, and so define a global smooth function on $X \backslash S$. We define

$$
\omega_{\mathrm{SRF}}=\omega_{0}+\sqrt{-1} \partial \bar{\partial} \rho
$$

which is a closed real $(1,1)$-form on $X \backslash S$, restricts to a Ricci-flat Kähler metric on all fibers $X_{y}$ of $y \in B \backslash S^{\prime}$.

Let $\Omega$ be the smooth volume form on $X$ with

$$
\sqrt{-1} \partial \bar{\partial} \log \Omega=\chi, \int_{X} \Omega=\left(\begin{array}{c}
n \\
m
\end{array}\right) \int_{X} \omega_{0}^{n-m} \wedge \chi^{m} .
$$

Define a function $F$ on $X \backslash S$ by

$$
F:=\frac{\Omega}{\left(\begin{array}{l}
n \\
m
\end{array}\right) \chi^{m} \wedge \omega_{\mathrm{SRF}}^{n-m}},
$$

then $F$ is constant along the fiber $X_{y}, y \in B \backslash S^{\prime}$, so it descends to a smooth function on $B \backslash S^{\prime}$. Then [7] showed that the Monge-Ampére equation

$$
(\chi+\sqrt{-1} \partial \bar{\partial} v)^{m}=F e^{v} \chi^{m},
$$

has a unique solution $v \in \operatorname{PSH}(\chi) \cap C^{0}(B) \cap C^{\infty}\left(B \backslash S^{\prime}\right)$. Define

$$
\omega_{B}=\chi+\sqrt{-1} \partial \bar{\partial} v
$$

which is a smooth Kähler metric on $B \backslash S^{\prime}$, satisfies the twisted KählerEinstein equation

$$
\operatorname{Ric}\left(\omega_{B}\right)=-\omega_{B}+\omega_{\mathrm{WP}},
$$

where $\omega_{\mathrm{WP}}$ is the smooth Weil-Petersson form on $B \backslash S^{\prime}$.

Now let $\omega=\omega(t)$ be the solution of the normalized Kähler-Ricci flow

$$
\frac{\partial}{\partial t} \omega=-\operatorname{Ric}(\omega)-\omega, \omega(0)=\omega_{0},
$$

which exists for all time. Define the reference metrics

$$
\hat{\omega}(t)=e^{-t} \omega_{0}+\left(1-e^{-t}\right) \chi,
$$

which are Kähler for all $t \geqslant 0$, and we can write $\omega(t)=\hat{\omega}(t)+\sqrt{-1} \partial \bar{\partial} \varphi(t)$, and $\varphi(0)=0$, then the Kähler-Ricci flow (2.4) is equivalent to the parabolic Monge-Ampére equation

$$
\frac{\partial}{\partial t} \varphi=\log \frac{e^{(n-m) t}(\hat{\omega}(t)+\sqrt{-1} \partial \bar{\partial} \varphi(t))^{n}}{\Omega}-\varphi, \varphi(0)=0 .
$$

From now on, we always set $K=f^{-1}\left(K^{\prime}\right)$ where $K^{\prime} \subset B \backslash S^{\prime}$ is a compact subset. Then we can choose some open subset $U^{\prime} \subset \subset B \backslash S^{\prime}$ such that $K^{\prime} \subset U^{\prime}$. Set $U=f^{-1}\left(U^{\prime}\right)$, then $K \subset \subset U \subset \subset X \backslash S$. Also, we denote by $h(t)$ some positive decreasing function on $[0, \infty)$ which tends to zero as $t \rightarrow \infty$. 
Now we have the following lemmas. See [13, 12, for unified discussions (and also [2, 7, 9]).

Lemma 2.1. There exists some constant $C=C(K)$ and $h(t)$ depending on the domain $K$, such that

(1) $C^{-1} \hat{\omega}(t) \leqslant \omega(t) \leqslant C \hat{\omega}(t)$, on $K \times[0, \infty)$.

(2) $|\varphi-v|+|\dot{\varphi}+\varphi-v| \leqslant h(t)$, on $K \times[0, \infty)$.

(3) There exists a uniform $C_{0}>0$ such that

$$
|R| \leqslant C_{0}, \text { on } X \times[0, \infty) \text {. }
$$

(4) $\operatorname{tr}_{\omega(t)} \omega_{B}-m \leqslant h(t)$, on $K \times[0, \infty)$.

(5) Especially, if $S=\emptyset$, then (1)-(4) hold with $K$ replaced by $X$ and $h(t)$ replaced by $C e^{-\eta t}$ for some constants $\eta, C>0$ depending on $\left(X, \omega_{0}\right)$.

Lemma 2.2. Along the normalized Kähler Ricci-flow, we have on $X \backslash S \times$ $[0, \infty)$

$$
\left(\frac{\partial}{\partial t}-\Delta\right)(\dot{\varphi}+\varphi-v)=\operatorname{tr}_{\omega(t)} \omega_{B}-m
$$

and there exists some $C=C(K)>0$ such that

$$
\left(\frac{\partial}{\partial t}-\Delta\right) \operatorname{tr}_{\omega(t)} \omega_{B} \leqslant C, \text { on } K \times[0, \infty) .
$$

Especially, when $S=\emptyset$, then (2.6), (2.7) holds on $X \times[0, \infty)$ with $C$ depending on $\left(X, \omega_{0}\right)$.

Next we define on $X \backslash S$ the reference metrics

$$
\widetilde{\omega}(t)=e^{-t} \omega_{\mathrm{SRF}}+\left(1-e^{-t}\right) \omega_{B} .
$$

Then we have the following theorem due to [13] (in the proof).

Theorem 2.3. There exists $h(t)$ depending on the domain $K$ such that

$$
\|\omega(t)-\widetilde{\omega}(t)\|_{C^{0}(K, \omega(t))} \leqslant h(t) .
$$

Especially, when $S=\emptyset$, then

$$
\|\omega(t)-\widetilde{\omega}(t)\|_{C^{0}(X, \omega(t))} \leqslant C e^{-\eta t} .
$$

for some constants $\eta, C>0$ depending on $\left(X, \omega_{0}\right)$.

We also need the following lemma to choose local coordinates on the regular part, see e.g. Lemma 5.6 of [12].

Lemma 2.4. Let $f: X^{n} \rightarrow Y^{m}$ be a holomorphic submersion between complex manifolds. Then given any point $x \in X$ we can find an open set $U \ni x$ and local holomorphic coordinates $\left(z_{1}, \ldots, z_{n}\right)$ on $U$ and $\left(y_{1}, \ldots, y_{m}\right)$ on $f(U)$ such that in these coordinates the map $f$ is given by $\left(z_{1}, \ldots, z_{n}\right) \mapsto$ $\left(z_{1}, \ldots, z_{m}\right)$, i.e., $y_{1}=z_{1}, \ldots, y_{m}=z_{m}$.

We can apply Lemma 2.4 to a point $x \in X \backslash S, y=f(x) \in B \backslash S^{\prime}$ to choose local coordinates, and we may call such coordinates "local product coordinates". 


\section{Convergence of the trace And norm of $\omega_{B}$ Along the Flow}

From now on, we denote by $T_{0}=\operatorname{tr}_{\omega(t)} \omega_{B}$.

In this section, we use Theorem 2.3 to prove $\left|T_{0}-m\right|+\left|\left\|\omega_{B}\right\|_{\omega(t)}^{2}-m\right| \rightarrow 0$ as $t \rightarrow \infty$ on $X \backslash S$. As before, we use $h(t), h_{1}(t), \ldots$ to denote positive decreasing functions on $[0,+\infty)$ which tends to zero as $t \rightarrow \infty$.

First, we have the following basic estimate.

Lemma 3.1. For any point $x \in U$ with local product coordinates given by Lemma 2.4 around $x$ and $y=f(x)$, say $\left(z_{1}, \ldots, z_{n}\right)$ around $x$ and $\left(y_{1}, \ldots, y_{m}\right)$ around $y$. Suppose on such coordinate neighborhood $\omega(t)$ is given by

$$
\omega(t)=\sum_{i, j=1}^{n} g(t)_{i \bar{j}} d z_{i} \wedge d \bar{z}_{j}
$$

then there exists some constant $C$ depending on the domain such that: for $1 \leqslant \alpha, \beta \leqslant m, m+1 \leqslant i, j \leqslant n$,

$$
\begin{gathered}
\left|g(t)_{\alpha \bar{\beta}}\right| \leqslant C,\left|g(t)_{\alpha \bar{j}}\right| \leqslant C e^{-\frac{t}{2}},\left|g(t)_{i \bar{j}}\right| \leqslant C e^{-t} . \\
\left|g(t)^{\alpha \bar{\beta}}\right| \leqslant C,\left|g(t)^{\alpha \bar{j}}\right| \leqslant C e^{\frac{t}{2}},\left|g(t)^{i \bar{j}}\right| \leqslant C e^{t} .
\end{gathered}
$$

at $x$. In particular, when $S=\emptyset$, (3.1) and (3.2) hold on $X \times[0, \infty)$ with $C$ depending on $\left(X, \omega_{0}\right)$.

Proof. We define on such coordinate neighborhood (contained in $U$ ) the local metrics

$$
\omega_{E}(t)=\omega^{(m)}+e^{-t} \omega^{(n-m)},
$$

where $\omega^{(m)}$ and $\omega^{(n-m)}$ denotes the standard Euclidean metrics on the two factors of $\mathbb{C}^{n}=\mathbb{C}^{m} \times \mathbb{C}^{n-m}$. Thanks to Lemma 2.1, we can find constant $C$ depending on the domain $K$ such that

$$
C^{-1} \omega_{E}(t) \leqslant \omega(t) \leqslant C \omega_{E}(t),
$$

Denote

$$
\omega_{B}=\sqrt{-1} \sum_{\alpha, \beta=1}^{m}\left(g_{B}\right)_{\alpha \bar{\beta}} d z_{\alpha} \wedge d \bar{z}_{\beta}, \omega_{\mathrm{SRF}}=\sqrt{-1} \sum_{i, j=1}^{n}\left(g_{\mathrm{SRF}}\right)_{i \bar{j}} d z_{i} \wedge d \bar{z}_{j}
$$

Hence using (2.8) of Theorem 2.3 we have

$$
\begin{aligned}
& C \geqslant\|\omega(t)-\widetilde{\omega}(t)\|_{\omega_{E}(t)}^{2} \\
& \geqslant \sum_{\alpha, \beta=1}^{m}\left|g(t)_{\alpha \bar{\beta}}-\left(1-e^{-t}\right)\left(g_{B}\right)_{\alpha \bar{\beta}}-e^{-t}\left(g_{\mathrm{SRF}}\right)_{\alpha \bar{\beta}}\right|^{2} \\
& +\sum_{\alpha=1}^{m} \sum_{j=m+1}^{n} e^{t}\left|g(t)_{\alpha \bar{j}}-e^{-t}\left(g_{\mathrm{SRF}}\right)_{\alpha \bar{j}}\right|^{2}+\sum_{i, j=m+1}^{n} e^{2 t}\left|g(t)_{i \bar{j}}-e^{-t}\left(g_{\mathrm{SRF}}\right)_{i \bar{j}}\right|^{2},
\end{aligned}
$$


on our coordinate neighborhood. Then we can apply the trivial inequality $|a-b|^{2} \geqslant \frac{1}{2}|a|^{2}-|b|^{2}$ to each term to conclude (3.1). Next, from Lemma 2.1. we have the first and third estimates of (3.2), and the second estimate then follows from Cauchy-Schwarz inequality.

Proposition 3.2. There exists $h(t)$ depending on $K$ such that

$$
\begin{gathered}
\left|T_{0}-m\right| \leqslant h(t), \text { on } K \times[0, \infty) . \\
\left|\left\|\omega_{B}\right\|_{\omega(t)}^{2}-m\right| \leqslant h(t), \text { on } K \times[0, \infty) .
\end{gathered}
$$

In particular, if $S=\emptyset$, then we have

$$
\left|T_{0}-m\right|+\left|\left\|\omega_{B}\right\|_{\omega(t)}^{2}-m\right| \leqslant C e^{-\eta t} \text {, on } X \times[0, \infty),
$$

where $\eta, C>0$ are constants depending on $\left(X, \omega_{0}\right)$.

Proof. Applying Theorem 2.3 to $\bar{U}$ there exists some $h_{1}(t)$ depending on the domain such that

$$
\|\omega(t)-\widetilde{\omega}(t)\|_{C^{0}(\bar{U}, \omega(t))}^{2} \leqslant h_{1}(t) .
$$

Now given any $x_{0} \in K$, we choose local product coordinate like Lemma 3.1, say $U_{0} \subset U$ around $x_{0}$. WLOG, we may assume

$$
U_{0}=B^{(m)}(1) \times B^{(n-m)}(1) \subset \mathbb{C}^{n}, f\left(U_{0}\right)=B^{(m)}(1) \subset \mathbb{C}^{m}, x_{0}=(0,0),
$$

where $B^{(m)}(1)$ and $B^{(n-m)}(1)$ denotes Euclidean unit balls in $\mathbb{C}^{m}$ and $\mathbb{C}^{n-m}$, respectively. The map $f$ is given by $f\left(z_{1}, \ldots, z_{n}\right)=\left(z_{1}, \ldots, z_{m}\right)$. Fix a time $t$, we define the transformation

$$
\begin{aligned}
F_{t}: & U_{1}:=B^{(m)}(1) \times B^{(n-m)}\left(e^{-\frac{t}{2}}\right) \rightarrow U_{0} \\
& F_{t}\left(w_{1}, \ldots, w_{n}\right)=\left(w_{1}, \ldots, w_{m}, e^{\frac{t}{2}} w_{m+1}, \ldots, e^{\frac{t}{2}} w_{n}\right),
\end{aligned}
$$

Then consider on $U_{1}$ the metrics

$$
\omega_{1}(t)=F_{t}^{*} \omega(t), \quad \widetilde{\omega}_{1}(t)=F_{t}^{*} \widetilde{\omega}(t) .
$$

We immediately have from (3.8) that

$$
\left\|\omega_{1}(t)-\widetilde{\omega}_{1}(t)\right\|_{\omega_{1}(t)}^{2}\left(x_{0}\right)=\|\omega(t)-\widetilde{\omega}(t)\|_{\omega(t)}^{2}\left(x_{0}\right) \leqslant h_{1}(t) .
$$

Denote on $U_{1}$

$$
\omega_{1}(t)=\sqrt{-1} \sum_{i, j=1}^{n} h(t)_{i \bar{j}} d w_{i} \wedge d \bar{w}_{j}, \widetilde{\omega}_{1}(t)=\sqrt{-1} \sum_{i, j=1}^{n} \widetilde{h}(t)_{i \bar{j}} d w_{i} \wedge d \bar{w}_{j},
$$


then by definition

$$
\begin{aligned}
& \omega_{1}(t)=F_{t}^{*}\left[\sqrt{-1} \sum_{i, j=1}^{n} g(t)_{i \bar{j}} d z_{i} \wedge d \bar{z}_{j}\right] \\
& =\sqrt{-1} \sum_{\alpha, \beta=1}^{m} g(t)_{\alpha \bar{\beta}} d w_{\alpha} \wedge d \bar{w}_{\beta}+2 R e\left[\sqrt{-1} \sum_{\alpha=1}^{m} \sum_{j=m+1}^{n} e^{\frac{t}{2}} g(t)_{\alpha \bar{j}} d w_{\alpha} \wedge d \bar{w}_{j}\right] \\
& \quad+\sqrt{-1} \sum_{i, j=m+1}^{n} e^{t} g(t)_{i \bar{j}} d w_{i} \wedge d \bar{w}_{j},
\end{aligned}
$$

hence we obtain: for $1 \leqslant \alpha, \beta \leqslant m, m+1 \leqslant i, j \leqslant n$

$$
\left\{\begin{array}{l}
h(t)_{\alpha \bar{\beta}}=g(t)_{\alpha \bar{\beta}}, h(t)^{\alpha \bar{\beta}}=g(t)^{\alpha \bar{\beta}}, \\
h(t)_{\alpha \bar{j}}=e^{\frac{t}{2}} g(t)_{\alpha \bar{j}}, h(t)^{\alpha \bar{j}}=e^{-\frac{t}{2}} g(t)^{\alpha \bar{j}}, \\
h(t)_{i \bar{j}}=e^{t} g(t)_{i \bar{j}}, h(t)^{i \bar{j}}=e^{-t} g(t)^{i \bar{j}}
\end{array}\right.
$$

Then we apply Lemma 3.1 to conclude there exists some constant $C=$ $C(K)>0$ such that

$$
\sum_{i, j=1}^{n}\left(\left|h(t)_{i \bar{j}}\right|+\left|h(t)^{i \bar{j}}\right|\right)\left(x_{0}\right) \leqslant C .
$$

Similarly, we have: for $1 \leqslant \alpha, \beta \leqslant m, m+1 \leqslant i, j \leqslant n$

$$
\left\{\begin{array}{l}
\widetilde{h}(t)_{\alpha \bar{\beta}}=\left(1-e^{-t}\right)\left(g_{B}\right)_{\alpha \bar{\beta}}+e^{-t}\left(g_{\mathrm{SRF}}\right)_{\alpha \bar{\beta}}, \\
\widetilde{h}(t)_{\alpha \bar{j}}=e^{-\frac{t}{2}}\left(g_{\mathrm{SRF}}\right)_{\alpha \bar{j}}, \\
\widetilde{h}(t)_{i \bar{j}}=\left(g_{\mathrm{SRF}}\right)_{i \bar{j}} .
\end{array}\right.
$$

Now at $x_{0}$ we define an $n \times n$ matrix

$$
A=\left(a_{i \bar{j}}\right)_{n \times n}=\left(\begin{array}{cc}
\left(g_{B}\right)_{\alpha \bar{\beta}}\left(x_{0}\right) & 0 \\
0 & \left(g_{\mathrm{SRF}}\right)_{i \bar{j}}\left(x_{0}\right)
\end{array}\right)_{1 \leqslant \alpha, \beta \leqslant m, m+1 \leqslant i, j \leqslant n} .
$$

We claim that

$$
\left\|\omega_{1}(t)-A\right\|_{\omega_{E}}^{2}\left(x_{0}\right) \leqslant h_{2}(t) .
$$

for some $h_{2}(t)$ depending on the domain, where $\omega_{E}$ is the standard Euclidean metric on $U_{1}$. To see this, we use (3.9) and (3.11) to obtain

$$
\begin{aligned}
C h_{1}(t) & \geqslant\left\|\omega_{1}(t)-\widetilde{\omega}_{1}(t)\right\|_{\omega_{E}}^{2}\left(x_{0}\right) \\
& =\sum_{i, j=1}^{n}\left|h(t)_{i \bar{j}}-\widetilde{h}(t)_{i \bar{j}}\right|^{2}\left(x_{0}\right) .
\end{aligned}
$$


But at $x_{0}$

$$
\begin{aligned}
\left\|\omega_{1}(t)-A\right\|_{\omega_{E}}^{2}= & \sum_{\alpha, \beta=1}^{m}\left|h(t)_{\alpha \bar{\beta}}-a_{\alpha \bar{\beta}}\right|^{2}+2 \sum_{\alpha=1}^{m} \sum_{j=m+1}^{n}\left|h(t)_{\alpha \bar{j}}\right|^{2} \\
& +\sum_{i, j=m+1}^{n}\left|h(t)_{i \bar{j}}-a_{i \bar{j}}\right|^{2} .
\end{aligned}
$$

Then we can use (3.12) and (3.14) to estimate three terms on the RHS of the above equality. Indeed, for $1 \leqslant \alpha, \beta \leqslant m$, we have at $x_{0}$

$$
\begin{aligned}
C h_{1}(t) & \geqslant\left|h(t)_{\alpha \bar{\beta}}-\widetilde{h}(t)_{\alpha \bar{\beta}}\right|^{2}=\left|h(t)_{\alpha \bar{\beta}}-\left(1-e^{-t}\right)\left(g_{B}\right)_{\alpha \bar{\beta}}-e^{-t}\left(g_{\mathrm{SRF}}\right)_{\alpha \bar{\beta}}\right|^{2} \\
& \geqslant \frac{1}{2}\left|h(t)_{\alpha \bar{\beta}}-a_{\alpha \bar{\beta}}\right|^{2}-e^{-2 t}\left|\left(g_{B}\right)_{\alpha \bar{\beta}}-\left(g_{\mathrm{SRF}}\right)_{\alpha \bar{\beta}}\right|^{2}
\end{aligned}
$$

which gives

$$
\sum_{\alpha, \beta=1}^{m}\left|h(t)_{\alpha \bar{\beta}}-a_{\alpha \bar{\beta}}\right|^{2} \leqslant 2 C h_{1}(t)+2 e^{-2 t}\left|\left(g_{B}\right)_{\alpha \bar{\beta}}-\left(g_{\mathrm{SRF}}\right)_{\alpha \bar{\beta}}\right|^{2} \leqslant h_{3}(t)
$$

Next, for $1 \leqslant \alpha \leqslant m, m+1 \leqslant j \leqslant n$, we have

$$
\begin{aligned}
C h_{1}(t) & \geqslant\left|h(t)_{\alpha \bar{j}}-\widetilde{h}(t)_{\alpha \bar{j}}\right|^{2}=\left|h(t)_{\alpha \bar{j}}-e^{-\frac{t}{2}}\left(g_{\mathrm{SRF}}\right)_{\alpha \bar{j}}\right|^{2} \\
& \geqslant \frac{1}{2}\left|h(t)_{\alpha \bar{j}}\right|^{2}-e^{-t}\left|\left(g_{\mathrm{SRF}}\right)_{\alpha \bar{j}}\right|^{2}
\end{aligned}
$$

which gives

$$
\sum_{\alpha=1}^{m} \sum_{j=m+1}^{n}\left|h(t)_{\alpha \bar{j}}\right|^{2} \leqslant 2 C h_{1}(t)+2 e^{-t}\left|\left(g_{\mathrm{SRF}}\right)_{\alpha \bar{j}}\right|^{2} \leqslant h_{4}(t)
$$

Finally, for $m+1 \leqslant i, j \leqslant n$, we have

$$
C h_{1}(t) \geqslant \sum_{i, j=m+1}^{n}\left|h(t)_{i \bar{j}}-\left(g_{\mathrm{SRF}}\right)_{i \bar{j}}\right|^{2}=\sum_{i, j=m+1}^{n}\left|h(t)_{i \bar{j}}-a_{i \bar{j}}\right|^{2},
$$

Combine the above three estimates we obtain (3.13) with

$$
h_{2}(t)=C h_{1}(t)+h_{3}(t)+h_{4}(t) .
$$


Now at $x_{0}$ we can use (3.11) and (3.13) to get

$$
\begin{aligned}
\mid & \operatorname{det} \omega_{1}(t)-\operatorname{det} A \mid \\
= & \left|\sum_{\left(j_{1}, \ldots, j_{n}\right)}(-1)^{\sigma\left(j_{1}, \ldots, j_{n}\right)}\left(h(t)_{1 \bar{j}_{1}} \cdots h(t)_{n \bar{j}_{n}}-a_{1 \bar{j}_{1}} \cdots a_{n \bar{j}_{n}}\right)\right| \\
\leqslant & \sum_{\left(j_{1}, \ldots, j_{n}\right)}\left|h(t)_{1 \bar{j}_{1}} \cdots h(t)_{(n-1) \bar{j}_{n-1}}\left(h(t)_{n \bar{j}_{n}}-a_{n \bar{j}_{n}}\right)\right|+ \\
& \ldots+\sum_{\left(j_{1}, \ldots, j_{n}\right)}\left|\left(h(t)_{1 \bar{j}_{1}}-a_{1 \bar{j}_{1}}\right) h(t)_{2 \bar{j}_{2}} \cdots h(t)_{n \bar{j}_{n}}\right| \\
\leqslant & \sum_{\left(j_{1}, \ldots, j_{n}\right)}\left(\left|h(t)_{1 \bar{j}_{1}}-a_{1 \bar{j}_{1}}\right|+\cdots+\left|h(t)_{n \bar{j}_{n}}-a_{n \bar{j}_{n}}\right|\right) \\
\leqslant & C h_{2}(t)^{\frac{1}{2}} .
\end{aligned}
$$

Hence we obtain

$$
\left|\operatorname{det} \omega_{1}(t)\left(x_{0}\right)-\operatorname{det} A\right| \leqslant h_{5}(t) .
$$

But det $A \in\left[A_{0}, A_{1}\right]$ for some positive constants $A_{0}, A_{1}$ depending on the domain, independent of $t$ (after $t$ is large), so we can choose a large time $T \geqslant 1$ such that for all $t \geqslant T$, we have

$$
\operatorname{det} \omega_{1}(t)\left(x_{0}\right) \in\left[\frac{1}{2} A_{0}, 2 A_{1}\right],
$$

Set $A^{-1}=\left(a^{i \bar{j}}\right)$. Now we use (3.11), (3.13), (3.15), (3.16) to estimate at $x_{0}$

$$
\begin{aligned}
& \left|h(t)^{1 \overline{1}}-a^{1 \overline{1}}\right| \\
& =\left|\sum_{\left(j_{2}, \cdots, j_{n}\right)}\left(\frac{(-1)^{\sigma\left(j_{2}, \cdots, j_{n}\right)} h(t)_{2 \bar{j}_{2}} \cdots h(t)_{n \bar{j}_{n}}}{\operatorname{det} \omega_{1}(t)}-\frac{(-1)^{\sigma\left(j_{2}, \cdots, j_{n}\right)} a_{2 \bar{j}_{2}} \cdots a_{n \bar{j}_{n}}}{\operatorname{det} A}\right)\right| \\
& \leqslant \frac{1}{\operatorname{det} \omega_{1}(t)} \sum_{\left(j_{2}, \cdots, j_{n}\right)}\left|h(t)_{2 \bar{j}_{2}} \cdots h(t)_{n \bar{j}_{n}}-a_{2 \bar{j}_{2}} \cdots a_{n \bar{j}_{n}}\right| \\
& \quad+\sum_{\left(j_{2}, \cdots, j_{n}\right)}\left|a_{2 \bar{j}_{2}} \cdots a_{n \bar{j}_{n}}\right|\left|\frac{1}{\operatorname{det} \omega_{1}(t)}-\frac{1}{\operatorname{det} A}\right| \\
& \leqslant \frac{1}{2} C h_{2}(t)^{\frac{1}{2}}+C \frac{h_{5}(t)}{\frac{1}{2} A_{0} \cdot A_{0}} \leqslant h_{6}(t),
\end{aligned}
$$

Similar argument holds for all $1 \leqslant i, j \leqslant n$, hence we obtain

$$
\sum_{i, j=1}^{n}\left|h(t)^{i \bar{j}}\left(x_{0}\right)-a^{i \bar{j}}\right| \leqslant h_{6}(t) .
$$


By the special form of the matrix $A$, we have

$$
\sum_{\alpha, \beta=1}^{m} a^{\alpha \bar{\beta}} a_{\alpha \bar{\beta}}=m, \quad \sum_{i, j, k, l=1}^{m} a^{i \bar{l}} a^{k \bar{j}} a_{i \bar{j}} a_{k \bar{l}}=m,
$$

hence we can apply (3.10), (3.11) and (3.17) to estimate at $x_{0}$

$$
\begin{aligned}
\left|T_{0}-m\right| & =\left|\sum_{\alpha, \beta=1}^{m} h(t)^{\alpha \bar{\beta}} a_{\alpha \bar{\beta}}-\sum_{\alpha, \beta=1}^{m} a^{\alpha \bar{\beta}} a_{\alpha \bar{\beta}}\right| \\
& \leqslant \sum_{\alpha, \beta=1}^{m}\left|h(t)^{\alpha \bar{\beta}}-a^{\alpha \bar{\beta}}\right| \cdot\left|a_{\alpha \bar{\beta}}\right| \leqslant h_{7}(t),
\end{aligned}
$$

which gives (3.5) at $x_{0}$, and similarly

$$
\begin{aligned}
& \left|\left\|\omega_{B}\right\|_{\omega(t)}^{2}-m\right|=\left|\sum_{i, j, k, l=1}^{m} h(t)^{i \bar{l}} h(t)^{k \bar{j}} a_{i \bar{j}} a_{k \bar{l}}-\sum_{i, j, k, l=1}^{m} a^{i \bar{l}} a^{k \bar{j}} a_{i \bar{j}} a_{k \bar{l}}\right| \\
& \leqslant \sum_{i, j, k, l=1}^{m}\left(\left|h(t)^{i \bar{l}}-a^{i \bar{l}}\right|\left|h(t)^{k \bar{j}} a_{i \bar{j}} a_{k \bar{l}}\right|+\left|h(t)^{i \bar{l}}\right|\left|h(t)^{k \bar{j}}-a^{k \bar{j}}\right|\left|a_{i \bar{j}} a_{k \bar{l}}\right|\right) \\
& \leqslant h_{8}(t),
\end{aligned}
$$

which gives (3.6) at $x_{0}$. Since $x_{0} \in K$ was arbitrary chosen, we obtain (3.5) and (3.6).

When $S=\emptyset$, the above estimates hold on the whole manifold $X$ with $h(t)$ replaced by $C e^{-\eta t}$ where $\eta, C>0$ are constants depending on $\left(X, \omega_{0}\right)$ which may change from line to line. This completes the proof.

\section{The PRoof of TheOrem 1.1}

In this section we prove Theorem 1.1. All the operators $\nabla, \Delta,\langle$,$\rangle are with$ respect to the evolving metric $\omega(t)$.

We first need the following basic lemma to improve our decreasing function $h(t)$.

Lemma 4.1. For any $h(t):[0, \infty) \rightarrow(0, \infty)$, a positive decreasing function which tends to zero as $t \rightarrow \infty$, there exists a smooth positive decreasing function $A(t):[0, \infty) \rightarrow(0, \infty)$ satisfying that: $h(t) \leqslant A(t), t \geqslant 0 ; A(t) \rightarrow 0$ as $t \rightarrow \infty$; and moreover

$$
0 \leqslant-A^{\prime}(t) \leqslant 100 A(t) \text {, on }[0, \infty) .
$$

Proof. Choose $0<\ell_{1}<\ell_{2}<\cdots$ in the following way: Let $\ell_{1} \gg 2$ such that $h\left(\ell_{1}\right)<\frac{1}{2} h(0)$. Then choose $\ell_{2} \gg \ell_{1}+2$ such that $h\left(\ell_{2}\right)<\frac{1}{2^{2}} h(0)$. Repeat this process, for each $k \geqslant 1$, we choose $\ell_{k+1} \gg \ell_{k}+2$ such that $h\left(\ell_{k+1}\right)<\frac{1}{2^{k+1}} h(0)$. First we define

$$
A(t) \equiv h(0), t \in\left[0, \ell_{1}\right] ; \quad A(t) \equiv \frac{1}{2^{k}} h(0), t \in\left[\ell_{k}+2, \ell_{k+1}\right], k \geqslant 1 .
$$


Hence for each $k \geqslant 1$, we have

$$
\left\{\begin{array}{l}
h(t) \leqslant h\left(\ell_{k}\right)<\frac{1}{2^{k}} h(0), t \in\left[\ell_{k}, \infty\right) \\
A(t) \equiv \frac{1}{2^{k-1}} h(0), t \in\left[\ell_{k}-1, \ell_{k}\right] \\
A(t) \equiv \frac{1}{2^{k}} h(0), t \in\left[\ell_{k}+2, \ell_{k}+3\right]
\end{array}\right.
$$

hence we can define $A(t)$ on $\left[\ell_{k}, \ell_{k}+2\right]$ such that $A(t)$ is smooth and decreasing on $\left[\ell_{k}-1, \ell_{k}+3\right]$ (and hence smooth and decreasing on $(0, \infty)$ ) and moreover

$$
0 \leqslant-A^{\prime}(t) \leqslant 200 \cdot \frac{\frac{1}{2^{k-1}} h(0)-\frac{1}{2^{k}} h(0)}{\left(\ell_{k}+2\right)-\ell_{k}}=\frac{100}{2^{k}} h(0) \leqslant 100 A(t),
$$

for $t \in\left[\ell_{k}, \ell_{k}+2\right]$. Outside such intervals, $A^{\prime}(t) \equiv 0$, hence (4.1) is verified. Also, on each $\left[\ell_{k}, \ell_{k+1}\right]$, we have

$$
h(t) \leqslant \frac{1}{2^{k}} h(0) \leqslant A(t)
$$

hence $h(t) \leqslant A(t)$ on $[0, \infty)$. Finally, $A(t) \rightarrow 0$ as $t \rightarrow \infty$ is easy to see. This finishes the proof.

Next, we need to construct local cutoff function.

Lemma 4.2. Recall that $K=f^{-1}\left(K^{\prime}\right), U=f^{-1}\left(U^{\prime}\right), K^{\prime} \subset \subset U^{\prime} \subset \subset B \backslash S^{\prime}$. Then there exists a smooth cutoff function $\rho$ with $\operatorname{supp}(\rho) \subset U, \rho>0$ on $U$, $0 \leqslant \rho \leqslant 1, \rho \equiv 1$ on $K$, satisfying

$$
|\nabla \rho|_{\omega(t)}^{2}+\left|\Delta_{\omega(t)} \rho\right| \leqslant C
$$

on $U \times[0, \infty)$ for some constant $C$ depending on the domain $K$.

Proof. We first choose cutoff function $\rho_{0}$ on $B$ such that $\operatorname{supp}\left(\rho_{0}\right) \subset U^{\prime}$, $\rho_{0}>0$ on $U^{\prime}, 0 \leqslant \rho_{0} \leqslant 1, \rho_{0} \equiv 1$ on $K^{\prime}$ and moreover

$$
\sqrt{-1} \partial \rho_{0} \wedge \bar{\partial} \rho_{0} \leqslant C \omega_{B}, \quad-C \omega_{B} \leqslant \sqrt{-1} \partial \bar{\partial} \rho_{0} \leqslant C \omega_{B},
$$

on $U^{\prime}$. Then we set $\rho=f^{*} \rho_{0}$. Using Lemma 2.1 and Lemma 3.1, we have under local product coordinates

$$
\begin{aligned}
& |\nabla \rho|_{\omega(t)}^{2}=\sum_{i, j=1}^{m} g(t)^{i \bar{j}} \partial_{i} \rho \partial_{\bar{j}} \rho \leqslant C, \\
& \left|\Delta_{\omega(t)} \rho\right|=\left|\operatorname{tr}_{\omega(t)} \sqrt{-1} \partial \bar{\partial} \rho\right| \leqslant C \operatorname{tr}_{\omega(t)} \omega_{B} \leqslant C,
\end{aligned}
$$

with some constant $C$ depending on the domain $K$. This finishes the proof.

In the following, we always use $h(t), A(t), B(t)$ to denote positive decreasing functions which tend to zero as $t \rightarrow \infty$, and moreover $A(t), B(t)$ satisfy condition (4.1). 
Set $u=\dot{\varphi}+\varphi-v$ on $X \backslash S$. Recall that $T_{0}=\operatorname{tr}_{\omega(t)} \omega_{B}$, and

$$
\left(\frac{\partial}{\partial t}-\Delta\right) u=T_{0}-m
$$

Then along the flow we have on $X \backslash S$

$$
\begin{gathered}
\left(\frac{\partial}{\partial t}-\Delta\right)|\nabla u|^{2}=|\nabla u|^{2}-|\nabla \nabla u|^{2}-|\nabla \bar{\nabla} u|^{2}+2 \operatorname{Re}\left(\nabla T_{0} \cdot \bar{\nabla} u\right) . \\
\left(\frac{\partial}{\partial t}-\Delta\right) \Delta u=\Delta u+\langle\operatorname{Ric}, \sqrt{-1} \partial \bar{\partial} u\rangle+\Delta T_{0} .
\end{gathered}
$$

We first estimate $|\nabla u|^{2}$.

Proposition 4.3. There exists $A(t)$ which depends on the domain $K$ such that

$$
|\nabla u|^{2} \leqslant A(t), \text { on } K \times[0, \infty) .
$$

In particular, when $S=\emptyset$, then we have

$$
|\nabla u|^{2} \leqslant C e^{-\eta t} \text {, on } X \times[0, \infty) .
$$

for some constants $\eta, C>0$ depending on $\left(X, \omega_{0}\right)$.

Proof. Apply Lemma 2.1 and Proposition [3.2, we can find some $h(t)$ depending on $K$ such that $|u|+\left|T_{0}-m\right| \leqslant h(t)$ on $\bar{U} \times[0, \infty)$. Then we choose $A(t)$ according to Lemma 4.1, such that $2 h(t) \leqslant A(t), t \geqslant 0$. Hence

$$
A(t)-u \in\left[\frac{1}{2} A(t), 2 A(t)\right],\left|T_{0}-m\right| \leqslant A(t), \text { on } \bar{U} \times[0, \infty) .
$$

So we can compute on $U \times[0, \infty)($ see [9])

$$
\begin{aligned}
& \left(\frac{\partial}{\partial t}-\Delta\right)\left(\frac{|\nabla u|^{2}}{A(t)-u}\right) \\
= & \frac{|\nabla u|^{2}-\left(|\nabla \nabla u|^{2}+|\nabla \bar{\nabla} u|^{2}\right)+2 R e\left(\nabla T_{0} \cdot \bar{\nabla} u\right)}{A(t)-u}-2 \epsilon \frac{\operatorname{Re}\left[\nabla|\nabla u|^{2} \cdot \bar{\nabla} u\right]}{(A(t)-u)^{2}} \\
& -2 \epsilon \frac{|\nabla u|^{4}}{(A(t)-u)^{3}}-\frac{2(1-\epsilon)}{A(t)-u} \operatorname{Re}\left[\nabla\left(\frac{|\nabla u|^{2}}{A(t)-u}\right) \cdot \bar{\nabla} u\right] \\
& +\left(T_{0}-m\right) \frac{|\nabla u|^{2}}{(A(t)-u)^{2}}-A^{\prime}(t) \frac{|\nabla u|^{2}}{(A(t)-u)^{2}},
\end{aligned}
$$

for any $\epsilon \in \mathbb{R}$. Now for some $k \in \mathbb{R}$ to be fixed, we can compute

$$
\left(\frac{\partial}{\partial t}-\Delta\right) \frac{1}{(A(t)-u)^{k}}=-\frac{k A^{\prime}(t)}{(A(t)-u)^{1+k}}-\frac{k(k+1)|\nabla u|^{2}}{(A(t)-u)^{2+k}}+\frac{k\left(T_{0}-m\right)}{(A(t)-u)^{1+k}},
$$


and

$$
\begin{aligned}
& \frac{2}{(A(t)-u)^{1+k}} \operatorname{Re}\left[\nabla\left(\frac{|\nabla u|^{2}}{A(t)-u}\right) \cdot \bar{\nabla} u\right] \\
& =\frac{2}{A(t)-u} \operatorname{Re}\left[\nabla\left(\frac{|\nabla u|^{2}}{(A(t)-u)^{1+k}}\right) \cdot \bar{\nabla} u\right]-2 k \frac{|\nabla u|^{4}}{(A(t)-u)^{3+k}},
\end{aligned}
$$

and hence on $U \times[0, \infty)$

$$
\begin{aligned}
& \left(\frac{\partial}{\partial t}-\Delta\right)\left(\frac{|\nabla u|^{2}}{(A(t)-u)^{1+k}}\right) \\
& =\frac{\left(\frac{\partial}{\partial t}-\Delta\right)\left(\frac{|\nabla u|^{2}}{A(t)-u}\right)}{(A(t)-u)^{k}}+\frac{|\nabla u|^{2}}{A(t)-u}\left(\frac{\partial}{\partial t}-\Delta\right) \frac{1}{(A(t)-u)^{k}} \\
& -2 R e\left[\nabla\left(\frac{|\nabla u|^{2}}{A(t)-u}\right) \cdot \bar{\nabla}\left(\frac{1}{(A(t)-u)^{k}}\right)\right] \\
& =\frac{|\nabla u|^{2}-\left(|\nabla \nabla u|^{2}+|\nabla \bar{\nabla} u|^{2}\right)+2 R e\left(\nabla T_{0} \cdot \bar{\nabla} u\right)}{(A(t)-u)^{1+k}}-2 \epsilon \frac{\operatorname{Re}\left[\nabla|\nabla u|^{2} \cdot \bar{\nabla} u\right]}{(A(t)-u)^{2+k}} \\
& -[2 \epsilon+k(k+1)-2 k(1-\epsilon+k)] \frac{|\nabla u|^{4}}{(A(t)-u)^{3+k}}+(1+k) \frac{\left(T_{0}-m\right)|\nabla u|^{2}}{(A(t)-u)^{2+k}} \\
& -\frac{2(1-\epsilon+k)}{A(t)-u} \operatorname{Re}\left[\nabla\left(\frac{|\nabla u|^{2}}{(A(t)-u)^{1+k}}\right) \cdot \bar{\nabla} u\right]-(1+k) \frac{A^{\prime}(t)|\nabla u|^{2}}{(A(t)-u)^{2+k}} .
\end{aligned}
$$

Now we set, in this proof, $k=-\frac{1}{3}, \epsilon=\frac{2}{3}$, then

$$
1-\epsilon+k=0,2 \epsilon+k(1+k)-2 k(1-\epsilon+k)=\frac{10}{9},
$$

hence (4.7) becomes

$$
\begin{aligned}
& \left(\frac{\partial}{\partial t}-\Delta\right)\left(\frac{|\nabla u|^{2}}{(A(t)-u)^{1+k}}\right) \\
= & \frac{|\nabla u|^{2}-\left(|\nabla \nabla u|^{2}+|\nabla \bar{\nabla} u|^{2}\right)+2 R e\left(\nabla T_{0} \cdot \bar{\nabla} u\right)}{(A(t)-u)^{1+k}}-\frac{4}{3} \cdot \frac{\operatorname{Re}\left[\nabla|\nabla u|^{2} \cdot \bar{\nabla} u\right]}{(A(t)-u)^{2+k}} \\
& -\frac{10}{9} \cdot \frac{|\nabla u|^{4}}{(A(t)-u)^{3+k}}+\frac{2}{3} \cdot \frac{\left(T_{0}-m\right)|\nabla u|^{2}}{(A(t)-u)^{2+k}}-\frac{2}{3} \cdot \frac{A^{\prime}(t)|\nabla u|^{2}}{(A(t)-u)^{2+k}} .
\end{aligned}
$$

We come to estimate each term. First, if we choose normal coordinates around a point in $U$, then we have

$\left.|\nabla| \nabla u\right|^{2} \cdot \bar{\nabla} u|=| u_{i}\left(u_{j} u_{\bar{j}}\right)_{\bar{i}}|=| u_{i} u_{j} u_{\overline{j i}}+\left.u_{i} u_{\bar{j}} u_{j \bar{i}}|\leqslant| \nabla u\right|^{2}(|\nabla \nabla u|+|\nabla \bar{\nabla} u|)$, 
hence

$$
\begin{aligned}
& \left|\frac{4}{3} \cdot \frac{R e\left[\nabla|\nabla u|^{2} \cdot \bar{\nabla} u\right]}{(A(t)-u)^{2+k}}\right| \\
& \leqslant 2\left(\frac{2}{3} \cdot \frac{|\nabla u|^{2}}{(A(t)-u)^{\frac{3+k}{2}}}\right)\left(\frac{|\nabla \nabla u|}{(A(t)-u)^{\frac{1+k}{2}}}+\frac{|\nabla \bar{\nabla} u|}{(A(t)-u)^{\frac{1+k}{2}}}\right) \\
& \leqslant \frac{|\nabla \nabla u|^{2}+|\nabla \bar{\nabla} u|^{2}}{(A(t)-u)^{1+k}}+\frac{8}{9} \cdot \frac{|\nabla u|^{4}}{(A(t)-u)^{3+k}},
\end{aligned}
$$

Next, since $k=-\frac{1}{3}$, we have $4+4 k=\frac{8}{3}=3+k$, hence

$$
\begin{aligned}
\frac{\left|2 R e\left(\nabla T_{0} \cdot \bar{\nabla} u\right)\right|}{(A(t)-u)^{1+k}} & \leqslant \frac{2\left|\nabla T_{0}\right| \cdot|\nabla u|}{(A(t)-u)^{1+k}} \leqslant\left|\nabla T_{0}\right|^{2}+\frac{|\nabla u|^{2}}{(A(t)-u)^{2+2 k}} \\
& \leqslant\left|\nabla T_{0}\right|^{2}+\frac{1}{100} \cdot \frac{|\nabla u|^{4}}{(A(t)-u)^{4+4 k}}+100 \\
& =\left|\nabla T_{0}\right|^{2}+\frac{1}{100} \cdot \frac{|\nabla u|^{4}}{(A(t)-u)^{3+k}}+100,
\end{aligned}
$$

The above two terms are the main terms that we need to be careful about, rest three terms are easy to control by using (4.1) and (4.6) (remember that $\left.k=-\frac{1}{3}\right)$ :

$$
\begin{aligned}
\frac{|\nabla u|^{2}}{(A(t)-u)^{1+k}} & \leqslant \frac{1}{100} \cdot \frac{|\nabla u|^{4}}{(A(t)-u)^{3+k}}+100(A(t)-u)^{1-k} \\
& \leqslant \frac{1}{100} \cdot \frac{|\nabla u|^{4}}{(A(t)-u)^{3+k}}+C \\
\left|\frac{2}{3} \cdot \frac{\left(T_{0}-m\right)|\nabla u|^{2}}{(A(t)-u)^{2+k}}\right| & \leqslant \frac{A(t)|\nabla u|^{2}}{\frac{1}{2} A(t) \cdot(A(t)-u)^{1+k}} \leqslant \frac{1}{100} \cdot \frac{|\nabla u|^{4}}{(A(t)-u)^{3+k}}+C, \\
\left|\frac{2}{3} \cdot \frac{A^{\prime}(t)|\nabla u|^{2}}{(A(t)-u)^{2+k}}\right| & \leqslant \frac{100 A(t)|\nabla u|^{2}}{\frac{1}{2} A(t) \cdot(A(t)-u)^{1+k}} \leqslant \frac{1}{100} \cdot \frac{|\nabla u|^{4}}{(A(t)-u)^{3+k}}+C,
\end{aligned}
$$

Hence we obtain on $U \times[0, \infty)$

$$
\left(\frac{\partial}{\partial t}-\Delta\right)\left(\frac{|\nabla u|^{2}}{(A(t)-u)^{1+k}}\right) \leqslant-\frac{1}{10} \cdot \frac{|\nabla u|^{4}}{(A(t)-u)^{3+k}}+\left|\nabla T_{0}\right|^{2}+C .
$$

Next, from Lemma 2.2 we have on $U \times[0, \infty)$

$$
\left(\frac{\partial}{\partial t}-\Delta\right) T_{0}^{2}=2 T_{0}\left(\frac{\partial}{\partial t}-\Delta\right) T_{0}-2\left|\nabla T_{0}\right|^{2} \leqslant C-2\left|\nabla T_{0}\right|^{2}
$$

Hence if we set

$$
Q=\frac{|\nabla u|^{2}}{(A(t)-u)^{1+k}}+T_{0}^{2},
$$

then we obtain from (4.9) and (4.10) that on $U \times[0, \infty)$

$$
\left(\frac{\partial}{\partial t}-\Delta\right) Q \leqslant-\frac{1}{10} \cdot \frac{|\nabla u|^{4}}{(A(t)-u)^{3+k}}+C .
$$


Now we choose cutoff function $\rho$ according to Lemma 4.2. Then we can compute

$$
\left(\frac{\partial}{\partial t}-\Delta\right)\left(\rho^{4} Q\right)=\rho^{4}\left(\frac{\partial}{\partial t}-\Delta\right) Q-Q \Delta \rho^{4}-2 R e\left[\nabla Q \cdot \bar{\nabla} \rho^{4}\right],
$$

For the second term, we use (4.2) to estimate on $U \times[0, \infty)$

$$
\begin{aligned}
-Q \Delta \rho^{4} & \leqslant C \rho^{2} Q=C \rho^{2} \frac{|\nabla u|^{2}}{(A(t)-u)^{1+k}}+C \rho^{2} T_{0}^{2} \\
& \leqslant \frac{1}{100} \cdot \frac{\rho^{4}|\nabla u|^{4}}{(A(t)-u)^{3+k}}+C(A(t)-u)^{1-k}+C \rho^{2} T_{0}^{2} \\
& \leqslant \frac{1}{100} \cdot \frac{\rho^{4}|\nabla u|^{4}}{(A(t)-u)^{3+k}}+C,
\end{aligned}
$$

For the third term, since $\rho>0$ on $U$, we have on $U \times[0, \infty)$

$$
\begin{aligned}
-2 \operatorname{Re}\left[\nabla Q \cdot \bar{\nabla} \rho^{4}\right] & =-2 \operatorname{Re}\left[\frac{\nabla\left(\rho^{4} Q\right)-4 \rho^{3} Q \nabla \rho}{\rho^{4}} \cdot 4 \rho^{3} \bar{\nabla} \rho\right] \\
& =-\frac{8}{\rho} \operatorname{Re}\left[\nabla\left(\rho^{4} Q\right) \cdot \bar{\nabla} \rho\right]+32 \rho^{2}|\nabla \rho|^{2} Q \\
& \leqslant-\frac{8}{\rho} \operatorname{Re}\left[\nabla\left(\rho^{4} Q\right) \cdot \bar{\nabla} \rho\right]+\frac{1}{100} \cdot \frac{\rho^{4}|\nabla u|^{4}}{(A(t)-u)^{3+k}}+C,
\end{aligned}
$$

hence combining (4.11) we obtain on $U \times[0, \infty)$

$$
\left(\frac{\partial}{\partial t}-\Delta\right)\left(\rho^{4} Q\right) \leqslant-\frac{1}{20} \cdot \frac{\rho^{4}|\nabla u|^{4}}{(A(t)-u)^{3+k}}-\frac{8}{\rho} \operatorname{Re}\left[\nabla\left(\rho^{4} Q\right) \cdot \bar{\nabla} \rho\right]+C
$$

Now, assume $\rho^{4} Q$ achieves its maximum at $\left(x_{0}, t_{0}\right)$ with $t_{0}>0$, then $x_{0} \notin \partial U$, hence $x_{0} \in U$ and then $\rho\left(x_{0}\right)>0$ and we have

$$
-\frac{8}{\rho} \operatorname{Re}\left[\nabla\left(\rho^{4} Q\right) \cdot \bar{\nabla} \rho\right]\left(x_{0}, t_{0}\right)=0
$$

Then we apply maximum principle to (4.12) to obtain

$$
0 \leqslant\left(\frac{\partial}{\partial t}-\Delta\right)\left(\rho^{4} Q\right)\left(x_{0}, t_{0}\right) \leqslant-\frac{1}{20} \cdot \frac{\rho^{4}|\nabla u|^{4}}{(A(t)-u)^{3+k}}\left(x_{0}, t_{0}\right)+C,
$$

which gives at $\left(x_{0}, t_{0}\right)$

$$
\frac{\rho^{4}|\nabla u|^{2}}{(A(t)-u)^{1+k}} \leqslant \frac{\rho^{4}|\nabla u|^{4}}{(A(t)-u)^{3+k}}+\rho^{4}(A(t)-u)^{1-k} \leqslant C .
$$

But $\rho^{4} T_{0}^{2} \leqslant C$ on $U \times[0, \infty)$, we conclude that $\rho^{4} Q \leqslant C$ on $U \times[0, \infty)$, which gives us that

$$
\frac{|\nabla u|^{2}}{(A(t)-u)^{\frac{2}{3}}} \leqslant C, \text { on } K \times[0, \infty),
$$

where $C$ is some constant depending on the domain $K$. Using (4.6) again, we obtain (4.5) with some larger $A(t)$. 
Finally, when $S=\emptyset$, the above arguments are still true on $X \times[0, \infty)$ with all $h(t)$ and $A(t)$ replaced by $C e^{-\eta t}$ with $\eta, C>0$ are constants depending on $\left(X, \omega_{0}\right)$ which may change from line to line, since its easy to see that

$$
\left|\frac{\left(C e^{-\eta t}\right)^{\prime}}{C e^{-\eta t}}\right|=\eta \leqslant 1
$$

(this is the motivation of Lemma 4.1). This completes the proof.

Now we come to estimate $|\Delta u|$ locally. We have the following proposition.

Proposition 4.4. There exists $A(t)$ depending on the domain $K$ such that

$$
|\Delta u| \leqslant A(t) \text {, on } K \times[0, \infty) .
$$

In particular, when $S=\emptyset$, then we have

$$
|\Delta u| \leqslant C e^{-\eta t}, \text { on } X \times[0, \infty) .
$$

for some constants $\eta, C>0$ depending on $\left(X, \omega_{0}\right)$.

Proof. Applying Lemma 2.1, Proposition 3.2 and Proposition 4.3, we can find $h(t)$ such that

$$
\left|T_{0}-m\right|+\left|\left\|\omega_{B}\right\|_{\omega(t)}^{2}-m\right|+|u|+|\nabla u|^{2} \leqslant \frac{1}{2} h(t), \text { on } \bar{U} \times[0, \infty),
$$

Then we apply Lemma 4.1 to find some $B(t)$ which then depends on the domain $K$ such that $h(t) \leqslant B(t)$ for $t \geqslant 0$ and $B(t)$ satisfies (4.1). WLOG, we may assume $B(t) \leqslant 1$ for $t \geqslant 0$, since otherwise we can consider $t \in[T, \infty)$ for some $T$ large. Now we set $A(t)=B(t)^{\frac{1}{2}} \geqslant B(t)$, then we have $A(t) \leqslant 1$ for $t \geqslant 0$ and moreover

$$
\left\{\begin{array}{l}
\left|T_{0}-m\right|+\left|\left\|\omega_{B}\right\|_{\omega(t)}^{2}-m\right|+|u| \leqslant \frac{1}{2} A(t), \\
|\nabla u|^{2} \leqslant A(t)^{2}
\end{array}\right.
$$

on $\bar{U} \times[0, \infty)$. Still, we have

$$
0 \leqslant-A^{\prime}(t)=-\frac{1}{2} \cdot \frac{B^{\prime}(t)}{B(t)^{\frac{1}{2}}} \leqslant \frac{1}{2} \cdot \frac{100 B(t)}{B(t)^{\frac{1}{2}}} \leqslant 100 B(t)^{\frac{1}{2}}=100 A(t),
$$

which means that $A(t)$ still satisfies (4.1).

Now we use (4.4) to compute

$$
\begin{aligned}
& \left(\frac{\partial}{\partial t}-\Delta\right)\left(\frac{-\Delta u}{A(t)-u}\right) \\
= & \frac{-\Delta u+|\nabla \bar{\nabla} u|^{2}+\left\langle\sqrt{-1} \partial \bar{\partial} u, \omega_{B}\right\rangle-\Delta T_{0}}{A(t)-u}-\frac{\left(T_{0}-m\right) \Delta u}{(A(t)-u)^{2}} \\
& -\frac{2}{A(t)-u} \operatorname{Re}\left[\nabla\left(\frac{-\Delta u}{A(t)-u}\right) \cdot \bar{\nabla} u\right]+\frac{A^{\prime}(t) \Delta u}{(A(t)-u)^{2}},
\end{aligned}
$$


which is always meaningful on $U \times[0, \infty)$ thanks to (4.14), where we have used the fact that on $X \backslash S \times[0, \infty)$

$$
\begin{aligned}
\operatorname{Ric}(\omega) & =-\sqrt{-1} \partial \bar{\partial}(\dot{\varphi}+\varphi)-\chi \\
& =-\sqrt{-1} \partial \bar{\partial}(\dot{\varphi}+\varphi-v)-(\chi+\sqrt{-1} \partial \bar{\partial} v) \\
& =-\sqrt{-1} \partial \bar{\partial} u-\omega_{B} .
\end{aligned}
$$

Also, we have

$$
\begin{aligned}
\left(\frac{\partial}{\partial t}-\Delta\right)\left(\frac{T_{0}-m}{A(t)-u}\right) & =\frac{\frac{\partial}{\partial t} T_{0}-\Delta T_{0}}{A(t)-u}-\frac{A^{\prime}(t)\left(T_{0}-m\right)}{(A(t)-u)^{2}} \\
& +\frac{\left(T_{0}-m\right)^{2}}{(A(t)-u)^{2}}-\frac{2}{A(t)-u} \operatorname{Re}\left[\nabla\left(\frac{T_{0}-m}{A(t)-u}\right) \cdot \bar{\nabla} u\right]
\end{aligned}
$$

Hence if we set

$$
K=\frac{-\Delta u-\left(T_{0}-m\right)}{A(t)-u},
$$

then we have

$$
\begin{aligned}
& \left(\frac{\partial}{\partial t}-\Delta\right) K \\
= & \frac{-\Delta u+|\nabla \bar{\nabla} u|^{2}+\left\langle\sqrt{-1} \partial \bar{\partial} u, \omega_{B}\right\rangle}{A(t)-u}-\frac{2}{A(t)-u} \operatorname{Re}[\nabla K \cdot \bar{\nabla} u] \\
& -\frac{\left(T_{0}-m\right) \Delta u}{(A(t)-u)^{2}}+\frac{A^{\prime}(t) \Delta u}{(A(t)-u)^{2}}-\frac{\frac{\partial}{\partial t} T_{0}}{A(t)-u}+\frac{A^{\prime}(t)\left(T_{0}-m\right)}{(A(t)-u)^{2}}-\frac{\left(T_{0}-m\right)^{2}}{(A(t)-u)^{2}},
\end{aligned}
$$

But using (4.16) we have on $X \backslash S$

$$
\begin{aligned}
\frac{\partial}{\partial t} T_{0} & =\frac{\partial}{\partial t}\left(g(t)^{i \bar{j}}\left(g_{B}\right)_{i \bar{j}}\right)=\left(R^{\bar{j} i}+g(t)^{i \bar{j}}\right)\left(g_{B}\right)_{i \bar{j}} \\
& =\left\langle\operatorname{Ric}, \omega_{B}\right\rangle+T_{0}=-\left\langle\sqrt{-1} \partial \bar{\partial} u, \omega_{B}\right\rangle+T_{0}-\left\|\omega_{B}\right\|_{\omega(t)}^{2},
\end{aligned}
$$

hence we obtain

$$
\begin{aligned}
& \left(\frac{\partial}{\partial t}-\Delta\right) K \\
= & \frac{-\Delta u+|\nabla \bar{\nabla} u|^{2}+2\left\langle\sqrt{-1} \partial \bar{\partial} u, \omega_{B}\right\rangle-T_{0}+\left\|\omega_{B}\right\|_{\omega(t)}^{2}}{A(t)-u} \\
& -\frac{2}{A(t)-u} R e[\nabla K \cdot \bar{\nabla} u]-\frac{\left(T_{0}-m\right) \Delta u}{(A(t)-u)^{2}}+\frac{A^{\prime}(t) \Delta u}{(A(t)-u)^{2}} \\
& +\frac{A^{\prime}(t)\left(T_{0}-m\right)}{(A(t)-u)^{2}}-\frac{\left(T_{0}-m\right)^{2}}{(A(t)-u)^{2}},
\end{aligned}
$$


Next, set $\epsilon=k=1$ in (4.7), we have (note that $A(t)$ is changed here)

$$
\begin{aligned}
& \left(\frac{\partial}{\partial t}-\Delta\right)\left(\frac{|\nabla u|^{2}}{(A(t)-u)^{2}}\right) \\
= & \frac{|\nabla u|^{2}-\left(|\nabla \nabla u|^{2}+|\nabla \bar{\nabla} u|^{2}\right)+2 R e\left(\nabla T_{0} \cdot \bar{\nabla} u\right)}{(A(t)-u)^{2}} \\
& -2 \frac{\operatorname{Re}\left[\nabla|\nabla u|^{2} \cdot \bar{\nabla} u\right]}{(A(t)-u)^{3}}-2 \frac{|\nabla u|^{4}}{(A(t)-u)^{4}}+\frac{2\left(T_{0}-m\right)|\nabla u|^{2}}{(A(t)-u)^{3}} \\
& -\frac{2 A^{\prime}(t)|\nabla u|^{2}}{(A(t)-u)^{3}}-\frac{2}{A(t)-u} \operatorname{Re}\left[\nabla\left(\frac{|\nabla u|^{2}}{(A(t)-u)^{2}}\right) \cdot \bar{\nabla} u\right] .
\end{aligned}
$$

Now set $H=\frac{|\nabla u|^{2}}{(A(t)-u)^{2}}$ and then set

$$
Q_{ \pm}= \pm K+100 H+T_{0}^{2}
$$

then combining (4.17) and (4.18) we obtain that on $U \times[0, \infty)$

$$
\begin{aligned}
& \left(\frac{\partial}{\partial t}-\Delta\right) Q_{ \pm} \\
= & \pm\left\{\frac{-\Delta u+|\nabla \bar{\nabla} u|^{2}+2\left\langle\sqrt{-1} \partial \bar{\partial} u, \omega_{B}\right\rangle-T_{0}+|| \omega_{B} \|_{\omega(t)}^{2}}{A(t)-u}\right. \\
& \left.-\frac{\left(T_{0}-m\right) \Delta u}{(A(t)-u)^{2}}+\frac{A^{\prime}(t) \Delta u}{(A(t)-u)^{2}}+\frac{A^{\prime}(t)\left(T_{0}-m\right)}{(A(t)-u)^{2}}-\frac{\left(T_{0}-m\right)^{2}}{(A(t)-u)^{2}}\right\} \\
& +100\left\{\frac{|\nabla u|^{2}-\left(|\nabla \nabla u|^{2}+|\nabla \bar{\nabla} u|^{2}\right)+2 R e\left(\nabla T_{0} \cdot \bar{\nabla} u\right)}{(A(t)-u)^{2}}\right. \\
& \left.-\frac{2 R e\left[\nabla|\nabla u|^{2} \cdot \bar{\nabla} u\right]}{(A(t)-u)^{3}}-\frac{2|\nabla u|^{4}}{(A(t)-u)^{4}}+\frac{2\left(T_{0}-m\right)|\nabla u|^{2}}{(A(t)-u)^{3}}-\frac{2 A^{\prime}(t)|\nabla u|^{2}}{(A(t)-u)^{3}}\right\} \\
- & \frac{2}{A(t)-u} \operatorname{Re}\left[\nabla Q_{ \pm} \cdot \bar{\nabla} u\right]+\frac{4 T_{0}}{A(t)-u} \operatorname{Re}\left(\nabla T_{0} \cdot \bar{\nabla} u\right) \\
+ & 2 T_{0}\left(\frac{\partial}{\partial t}-\Delta\right) T_{0}-2\left|\nabla T_{0}\right|^{2} .
\end{aligned}
$$

With the help of (4.14), we only need two "good terms"

$$
-100 \frac{|\nabla \nabla u|^{2}+|\nabla \bar{\nabla} u|^{2}}{(A(t)-u)^{2}}, \quad-2\left|\nabla T_{0}\right|^{2},
$$

to control all other terms except the term which involves $\nabla Q_{ \pm}$. Indeed, we have on $U \times[0, \infty)$

$$
\begin{aligned}
& \left|\frac{\Delta u}{A(t)-u}\right| \leqslant \frac{C|\nabla \bar{\nabla} u|}{A(t)-u} \leqslant \frac{|\nabla \bar{\nabla} u|^{2}}{(A(t)-u)^{2}}+C ; \\
& \frac{|\nabla \bar{\nabla} u|^{2}}{(A(t)-u)} \leqslant \frac{2 A(t)|\nabla \bar{\nabla} u|^{2}}{(A(t)-u)^{2}} \leqslant 2 \frac{|\nabla \bar{\nabla} u|^{2}}{(A(t)-u)^{2}}
\end{aligned}
$$




$$
\begin{gathered}
\left|\frac{2\left\langle\sqrt{-1} \partial \bar{\partial} u, \omega_{B}\right\rangle}{A(t)-u}\right| \leqslant \frac{2|\nabla \bar{\nabla} u| \cdot\left\|\omega_{B}\right\|_{\omega(t)}}{A(t)-u} \leqslant \frac{|\nabla \bar{\nabla} u|^{2}}{(A(t)-u)^{2}}+C ; \\
\left|\frac{T_{0}-|| \omega_{B} \|_{\omega(t)}^{2}}{A(t)-u}\right| \leqslant \frac{\left|T_{0}-m\right|+||\left|\omega_{B} \|_{\omega(t)}^{2}-m\right|}{A(t)-u} \leqslant \frac{\frac{1}{2} A(t)+\frac{1}{2} A(t)}{\frac{1}{2} A(t)}=2 ; \\
\left|\frac{\left(T_{0}-m\right) \Delta u}{(A(t)-u)^{2}}\right| \leqslant \frac{A(t) \cdot C|\nabla \bar{\nabla} u|}{(A(t)-u)^{2}} \leqslant \frac{|\nabla \bar{\nabla} u|^{2}}{(A(t)-u)^{2}}+C ; \\
\left|\frac{A^{\prime}(t) \Delta u}{(A(t)-u)^{2}}\right| \leqslant \frac{100 A(t) \cdot C|\nabla \bar{\nabla} u|}{(A(t)-u)^{2}} \leqslant \frac{|\nabla \bar{\nabla} u|^{2}}{(A(t)-u)^{2}}+C ; \\
\left|\frac{A^{\prime}(t)\left(T_{0}-m\right)}{(A(t)-u)^{2}}-\frac{\left(T_{0}-m\right)^{2}}{(A(t)-u)^{2}}\right| \leqslant C \\
100 \frac{|\nabla u|^{2}}{(A(t)-u)^{2}} \leqslant 100 \frac{A(t)^{2}}{\frac{1}{4} A(t)^{2}} \leqslant C ; \\
\left|\frac{2 R e\left(\nabla T_{0} \cdot \bar{\nabla} u\right)}{A(t)-u}\right| \leqslant \frac{2\left|\nabla T_{0}\right| \cdot A(t)}{\frac{1}{2} A(t)} \leqslant\left|\nabla T_{0}\right|^{2}+C ;
\end{gathered}
$$

and moreover

$$
\begin{aligned}
& 100\left\{-\frac{2 R e\left[\nabla|\nabla u|^{2} \cdot \bar{\nabla} u\right]}{(A(t)-u)^{3}}+\frac{2\left(T_{0}-m\right)|\nabla u|^{2}}{(A(t)-u)^{3}}-\frac{2 A^{\prime}(t)|\nabla u|^{2}}{(A(t)-u)^{3}}\right\} \\
& \leqslant C\left\{\frac{A(t)^{2}(|\nabla \nabla u|+|\nabla \bar{\nabla} u|)}{\frac{1}{4} A(t)^{2} \cdot(A(t)-u)}+\frac{A(t)^{3}}{A(t)^{3}}\right\} \\
& \leqslant \frac{|\nabla \nabla u|^{2}+|\nabla \bar{\nabla} u|^{2}}{(A(t)-u)^{2}}+C ; \\
& \frac{4 T_{0}}{A(t)-u} \operatorname{Re}\left(\nabla T_{0} \cdot \bar{\nabla} u\right)+2 T_{0}\left(\frac{\partial}{\partial t}-\Delta\right) T_{0} \leqslant\left|\nabla T_{0}\right|^{2}+C ;
\end{aligned}
$$

Hence we conclude that on $U \times[0, \infty)$

$$
\left(\frac{\partial}{\partial t}-\Delta\right) Q_{ \pm} \leqslant-5 \frac{|\nabla \bar{\nabla} u|^{2}}{(A(t)-u)^{2}}-\frac{2}{A(t)-u} \operatorname{Re}\left[\nabla Q_{ \pm} \cdot \bar{\nabla} u\right]+C .
$$

Now as before, we choose cutoff function $\rho$ according to Lemma 4.2, then we have that on $U \times[0, \infty)$

$$
\begin{aligned}
& \left(\frac{\partial}{\partial t}-\Delta\right)\left(\rho^{4} Q_{ \pm}\right) \\
& \leqslant-5 \frac{\rho^{4}|\nabla \bar{\nabla} u|^{2}}{(A(t)-u)^{2}}-\frac{2 \rho^{4} R e\left[\nabla Q_{ \pm} \cdot \bar{\nabla} u\right]}{A(t)-u}+C-Q_{ \pm} \Delta \rho^{4}-2 R e\left[\nabla Q_{ \pm} \cdot \bar{\nabla} \rho^{4}\right]
\end{aligned}
$$


For the forth term, we have

$$
\begin{aligned}
-Q_{ \pm} \Delta \rho^{4} & \leqslant C \rho^{2}\left|Q_{ \pm}\right| \\
& \leqslant C \rho^{2}\left\{\frac{|\nabla \bar{\nabla} u|}{A(t)-u}+\frac{\left|T_{0}-m\right|}{A(t)-u}+100 \frac{|\nabla u|^{2}}{(A(t)-u)^{2}}+T_{0}^{2}\right\} \\
& \leqslant \frac{\rho^{4}|\nabla \bar{\nabla} u|^{2}}{(A(t)-u)^{2}}+C
\end{aligned}
$$

for the second term, we have

$$
\begin{aligned}
& -\frac{2 \rho^{4} \operatorname{Re}\left[\nabla Q_{ \pm} \cdot \bar{\nabla} u\right]}{A(t)-u} \\
& =-\frac{2}{A(t)-u} \operatorname{Re}\left[\nabla\left(\rho^{4} Q_{ \pm}\right) \cdot \bar{\nabla} u\right]+\frac{8 \rho^{3} Q_{ \pm}}{A(t)-u} \operatorname{Re}[\nabla \rho \cdot \bar{\nabla} u] \\
& \leqslant-\frac{2}{A(t)-u} \operatorname{Re}\left[\nabla\left(\rho^{4} Q_{ \pm}\right) \cdot \bar{\nabla} u\right]+\frac{C \rho^{3}\left|Q_{ \pm}\right| A(t)^{2}}{A(t)} \\
& \leqslant-\frac{2}{A(t)-u} \operatorname{Re}\left[\nabla\left(\rho^{4} Q_{ \pm}\right) \cdot \bar{\nabla} u\right]+\frac{\rho^{4}|\nabla \bar{\nabla} u|^{2}}{(A(t)-u)^{2}}+C
\end{aligned}
$$

and similarly for the last term

$$
-2 \operatorname{Re}\left[\nabla Q_{ \pm} \cdot \bar{\nabla} \rho^{4}\right] \leqslant-\frac{8}{\rho} \operatorname{Re}\left[\nabla\left(\rho^{4} Q_{ \pm}\right) \cdot \bar{\nabla} \rho\right]+\frac{\rho^{4}|\nabla \bar{\nabla} u|^{2}}{(A(t)-u)^{2}}+C .
$$

Hence we finally conclude on $U \times[0, \infty)$

$$
\begin{aligned}
& \left(\frac{\partial}{\partial t}-\Delta\right)\left(\rho^{4} Q_{ \pm}\right) \\
& \leqslant-\frac{\rho^{4}|\nabla \bar{\nabla} u|^{2}}{(A(t)-u)^{2}}-\frac{2 R e\left[\nabla\left(\rho^{4} Q_{ \pm}\right) \cdot \bar{\nabla} u\right]}{A(t)-u}-\frac{8}{\rho} \operatorname{Re}\left[\nabla\left(\rho^{4} Q_{ \pm}\right) \cdot \bar{\nabla} \rho\right]+C .
\end{aligned}
$$

Now we assume $\rho^{4} Q_{+}$achieves its maximum at $\left(x_{0}, t_{0}\right)$ with $t_{0}>0$, then if $x_{0} \in \partial U$, we are done. Hence we can assume that $x_{0} \in U$ and then $\rho\left(x_{0}\right)>0$ and hence

$$
-\frac{2 R e\left[\nabla\left(\rho^{4} Q_{+}\right) \cdot \bar{\nabla} u\right]}{A(t)-u}\left(x_{0}, t_{0}\right)-\frac{8}{\rho} \operatorname{Re}\left[\nabla\left(\rho^{4} Q_{+}\right) \cdot \bar{\nabla} \rho\right]\left(x_{0}, t_{0}\right)=0,
$$

then we obtain from maximum principle and (4.20) that

$$
0 \leqslant\left(\frac{\partial}{\partial t}-\Delta\right)\left(\rho^{4} Q_{+}\right)\left(x_{0}, t_{0}\right) \leqslant-\frac{\rho^{4}|\nabla \bar{\nabla} u|^{2}}{(A(t)-u)^{2}}\left(x_{0}, t_{0}\right)+C,
$$


and hence on $U \times[0, \infty)$

$$
\begin{aligned}
\rho^{4} Q_{+} & \leqslant \rho^{4} Q_{+}\left(x_{0}, t_{0}\right) \\
& =\rho^{4}\left\{-\frac{\Delta u+\left(T_{0}-m\right)}{A(t)-u}+100 \frac{|\nabla u|^{2}}{(A(t)-u)^{2}}+T_{0}^{2}\right\}\left(x_{0}, t_{0}\right) \\
& \leqslant \frac{\rho^{4}|\nabla \bar{\nabla} u|}{A(t)-u}\left(x_{0}, t_{0}\right)+C \leqslant C,
\end{aligned}
$$

which gives

$$
\frac{-\Delta u}{A(t)-u} \leqslant C, \text { on } K \times[0, \infty)
$$

Similarly, consider $Q_{-}$instead gives us

$$
\frac{\Delta u}{A(t)-u} \leqslant C, \text { on } K \times[0, \infty)
$$

and hence we conclude

$$
|\Delta u| \leqslant A(t), \text { on } K \times[0, \infty),
$$

for some larger $A(t)$. Hence we obtain (4.13).

Finally, when $S=\emptyset$, the above arguments are still true on $X \times[0, \infty)$ with all $h(t)$ and $A(t)$ replaced by $C e^{-\eta t}$ with $\eta, C>0$ are constants depending on $\left(X, \omega_{0}\right)$ which may change from line to line. This completes the proof.

Now we can prove Theorem 1.1.

Proof of Theorem 1.1. From (4.16), we have that on $X \backslash S \times[0, \infty)$

$$
R=-T_{0}-\Delta u,
$$

hence Proposition 3.2 and Proposition 4.4 give us some $h(t)$ depending on the domain $K$ such that

$$
|R+m| \leqslant\left|T_{0}-m\right|+|\Delta u| \leqslant h(t) \text {, on } K \times[0, \infty) .
$$

In particular, if $S=\emptyset$, then

$$
|R+m| \leqslant\left|T_{0}-m\right|+|\Delta u| \leqslant C e^{-\eta t}, \text { on } X \times[0, \infty),
$$

where $\eta, C>0$ are constants depending on $\left(X, \omega_{0}\right)$. This completes the proof.

\section{REFERENCES}

[1] Aubin, T., Équations du type Monge-Ampère sur les variétés kähleriennes compactes, C. R. Acad. Sci. Paris Sér. A-B 283 (1976), no. 3, Aiii, A119-A121.

[2] Fong, F. T.-H., Zhang, Z., The collapsing rate of the Kähler-Ricci flow with regular infinite time singularity, J. reine angew. Math. 703 (2015), 95-113.

[3] G. Edwards, A scalar curvature bound along the conical Kähler-Ricci flow, J. Geom. Anal., 2017.DOI:10.1007/s12220-017-9817-0.

[4] Jian, W., Shi, Y., Song, J., A remark on constant scalar curvature Kähler metrics on minimal models, arXiv:1805.06863v1.

[5] Sesum, N., Tian, G., Bounding scalar curvature and diameter along the Kähler Ricci flow (after Perelman), J. Inst. Math. Jussieu 7 (2008), no. 3, 575-587. 
[6] Song, J., Tian, G., The Kähler-Ricci flow on surfaces of positive Kodaira dimension, Invent. Math. 170 (2007), no. 3, 609-653.

[7] Song, J., Tian, G., Canonical measures and Kähler-Ricci flow, J. Amer. Math. Soc., 25 (2012), no. 2, 303-353.

[8] Song, J., Tian, G., The Kähler-Ricci flow through singularities, Invent. Math. 207 (2017), 519-595.

[9] Song, J., Tian, G., Bounding scalar curvature for global solutions of the Kähler-Ricci flow, Amer. J. Math. 138 (2016), no. 3, 683-695.

[10] Song, J., Weinkove, B., Introduction to the Kähler-Ricci flow, Chapter 3 of 'Introduction to the Kähler-Ricci flow', eds S. Boucksom, P. Eyssidieux, V. Guedj, Lecture Notes Math. 2086, Springer 2013.

[11] Tian, G. and Zhang, Z., On the Kähler-Ricci flow on projective manifolds of general type, Chinese Ann. Math. Ser. B 27 (2006), no. 2, 179-192

[12] Tosatti, V., KAWA lecture notes on the Kähler-Ricci flow, arXiv:1508.04823

[13] Tosatti, V., Weinkove, B., and Yang, X.K., The Kähler-Ricci flow, Ricci-flat metrics and collapsing limits, arXiv:1408.0161, to appear in Amer. J. Math.

[14] Tosatti, V., Y.G. Zhang, Infinite time singularities of the Kähler-Ricci flow, Geom. Topol., 19 (2015), 2925-2948.

[15] Tsuji, H., Existence and degeneration of Kähler-Einstein metrics on minimal algebraic varieties of general type, Math. Ann. 281 (1988), no. 1, 123-133.

[16] Yau, S.-T., On the Ricci curvature of a compact Kähler manifold and the complex Monge-Ampère equation, I, Comm. Pure Appl. Math. 31 (1978), 339-411.

[17] Zhang, Z., Scalar curvature bound for Kähler-Ricci flows over minimal manifolds of general type, Int. Math. Res. Not. 2009; doi: 1093/imrn/rnp073

[18] Zhang, Z., Scalar curvature behavior for finite time singulairty of Kähler-Ricci flow,Michigan Math. J. 59 (2010), no. 2, 419-433

School of Mathematical Sciences, Peking University, Yiheyuan Road 5,BeiJING, P.R.CHINA, 100871

E-mail address: 1401110008@pku.edu.cn 